\title{
Discours anthropologique et littérature
}

\author{
Philippe Daros \\ Université Sorbonne Nouvelle - Paris $3^{*}$
}

L'action de la littérature peut être définie comme une réflexion sur la question de l'identité du sujet historique dans sa représentation fictionnelle sous la figure du personnage, de ses actions, de son rapport à l'espace, au temps, de ses pratiques symboliques dans des termes similaires à l'approche anthropologique contemporaine de l'identité conçue en termes d'identification et de relation. C'est cette conception anthropologique du fait littéraire qui sera examinée dans une perspective de critique littéraire.

Mots-clés : littérature, anthropologie, sciences humaines, critique littéraire, sujet, identité.

\section{Généalogie d’une «Querelle de propriété »}

L'expression est de Ramon Fernandez et il la formule, en 1935, pour qualifier les rapports entre sciences humaines, sociales et littérature. Vingt ans plus tard, Claude Lévi-Strauss publie Tristes Tropiques et ce « roman ethnologique » connaît un retentissement considérable dans la presse, dans la critique littéraire. Raymond Aron, dans Libération, en date du 16 novembre loue l'ouvrage mais s'interroge pour savoir à quel genre il appartient. Toujours est-il que tous les intellectuels de l'époque de Claude Roy à Georges Bataille, de Pierre Nora à Maurice Blanchot, rendent compte de cet « événement littéraire ». Tous sauf un : Roland Barthes, alors pourtant au cœur des grands débats intellectuels sur la littérature depuis la parution du Degré zéro de l'écriture (1953), demeure totalement silencieux ; ne disant absolument rien de cet essai entre littérature et document ethnographique. Ce silence est extrêmement significatif d'une opposition née plus d'un siècle auparavant, d'une querelle de territoire entre discours « scientifique » ethnologique et fait littéraire. D'entrée de jeu, en effet, nous le verrons, les rapports entre cette science humaine et la littérature ont témoigné simultanément, de la part des écrivains, d'une forte fascination, mais aussi de méfiance, voire d'un rejet.

*philippe.daros@univ-paris3.fr 
Je fais volontiers, avec Vincent Debaene à qui je dois toutes les informations qui précèdent extraites de son ouvrage L'adieu au voyage. L'ethnologie française entre science et littérature (Debaene 2010), l'hypothèse que Barthes a dû détester ce livre à cause de son écriture « bourgeoise », rhétorique, marquée par un régime anthropomorphique de l'image, ce régime que dénoncera, pour des motifs poétiques, idéologiques, anthropologiques, Alain Robbe-Grillet quelques années plus tard dans son essai polémique Pour un nouveau roman (Robbe-Grillet 1963). Chacun le sait aujourd'hui, l'époque était alors à la déconstruction du grand roman « aristotélicien » du XIX ${ }^{\mathrm{e}}$ siècle pour d'évidentes raisons, théoriques (rôle décisif du formalisme russe), idéologiques (l'héritage critique de "l'ère du soupçon ») et peut-être de façon encore plus fondamentalement historique que ne l'explicite Nathalie Sarraute : comme conséquence d'un refoulement de l'histoire récente, de l'histoire de la Catastrophe et de sa conséquence la plus marquante, la plus irréversible, celle d'une remise en cause de l'anthropogenèse de l'humanisme héritée des Lumières, de la tension kantienne vers les Lumières. Cette déconstruction, ce devenir métaphorique des valeurs de l'Occident, a eu pour conséquence un refus du roman centré sur la psychologie comportementale, autour d'une saisie « interne » de la part d'un auteur clairvoyant, de l'identité fictionnelle au profit de cette « mythologie de l'écriture » qui perdurera tout au long des années 70, en France tout au moins. C'est donc en suivant l'affirmation péremptoire, marque d'une époque aujourd'hui partiellement révolue, d'une certaine « mort de l'homme » que Barthes fait silence sur l'humanisme, pour lui, à la fois déphasé et politiquement réactionnaire, dont porte preuve Tristes Tropiques.

Mais ce n'est là que la phase historique la plus récente de déconstruction de l'humanisme que j'évoque et, donc, d'occultation de la question, anthropologique entre toutes, celles du sujet et de ses relations au monde historique.

Depuis bien longtemps, les rapports entre discours « scientifique » et fait littéraire ont été un enjeu essentiel, dans une définition réflexive de ce fait littéraire. Si l'on en parle de façon très générale, il est évident que la littérature, l'art dans son ensemble ont toujours été liés à une saisie descriptive de la question du sujet, et de ses représentations. Dans une finalité claire : rendre compte des conduites humaines par rapport au $\mathrm{B} / \mathrm{bien}$, au vrai (ou au « vraisemblable »), à la morale, à l'éthique etc. D'un point de vue poétique, le roman mais aussi le théâtre vont, conformément d'ailleurs à un certain idéal des Lumières exalter l'individu, le personnage romanesque dans sa singularité, dans son aptitude à s'affirmer comme « je » dans son agir et les répercussions de cette action par rapport aux autres, à la société, d'où, bien entendu, la nature en quelque manière « héroïque » de ce personnage, dont Lotman disait qu'il apparaît dans la fiction romanesque comme celui qui « troue » un champ sémantique. Et c'est là la raison - qu'analyse longuement Marielle Macé dans son ouvrage récent Styles. Critique de nos formes de vie (Macé 2016) -, de l'apparition, au XIXe siècle, de la notion 
de « style », de la saisie narrative des comportements dont l'œuvre de Baudelaire sera un paradigme avec sa figure du « dandy », de la volonté de différence. Car, dit Marielle Macé en préambule de sa réflexion : « ... la question du style [...] engage l'inlassable conquête de valeur rejouée en toute forme. Le « style » en cela ne s'oppose ni au banal, ni au commun, mais à l'indifférence. C'est d'ailleurs la leçon de la littérature, dans son immanence : toute singularité compte, car elle peut être l'amorce d'un possible de la vie. » (Macé 2016: 19-20).

Cette exaltation de la singularité du personnage dans son « milieu » explique, bien entendu, l'intérêt porté à « la psychologie » par la littérature de ce $\mathrm{XIX}^{\mathrm{e}}$ siècle, une littérature qui voit dans la peinture de l'homme « tout entier » sa fonction cardinale et sa spécificité, fonction au demeurant affirmée à l'époque, par Hugo : « Vous savez que je ne dédaigne aucun détail et que pour moi, tout ce qui touche à l'homme révèle l'homme. J'examine l'habit comme j'étudie l'édifice. Le costume est le premier vêtement de l'homme, la maison est le second. » (Hugo 1842 : 198, cité par Marielle Macé, 2016 : 168). D’où, bien entendu encore, le rôle qui sera conféré par les Naturalistes au " milieu », aux déterminismes génétiques, etc. La littérature est là pour rendre compte de ces singularités comportementales mais plus encore, dans cette tradition naturaliste, pour les rendre, dans l'affirmation de la toute puissance herméneutique de «l'auteur », lisibles, dicibles et ce, dans un but peu ou prou lié à une morale, ou contre une morale dominante.

Mais c'est à partir du développement intense des sciences positives, au XIX ${ }^{\mathrm{e}}$ que naît donc une « querelle de propriété », en forme de conflit où la littérature se sent dépossédée de son objet, de ce que Bataille, commentant, un siècle plus tard, Tristes Tropiques avait qualifié du propre de la littérature : une recherche « qui "engage l'homme tout entier", [...] " "la dimension de l'homme", tout ce qui "rend sensible un élément souverain, que ne subordonne aucun calcul" » (Bataille 1956 ; cité par Vincent Debaene, 2010). C'est, au demeurant, autour de cette expression « sans calcul », c'est-à-dire sans limitation logique, sans réduction scientiste à un savoir explicatif, au « calcul » que se nouera le conflit entre anthropologie, entre les sciences humaines en général, et la littérature.

\section{Épistémologie d'un grand partage}

Il importe en effet de rappeler qu'une grande ligne de partage se met en place depuis le début du XIX ${ }^{\mathrm{e}}$ siècle, entre les sciences « positives » et la littérature au sujet de leur fonctions respectives : rendre compte de la question du «Qui ? »1', de la « dimension de l'homme».

\footnotetext{
${ }^{1}$ C'est là un constat que retrouve Gisèle Sapiro (2011), lorsqu'elle évoque l'apparition de « discours d'expertises »: « ... les écrivains se trouvent, dès le milieu du $\mathrm{XIX}^{\mathrm{e}}$ siècle, en concurrence avec les nouveaux groupes professionnels qui interviennent au nom de leur expertise - c'est le cas notamment des nouvelles sciences sociales : statisticiens, démographes, économistes, sociologues. $\mathrm{Ne}$ détenant aucun savoir positif, ils se trouvent fragilisés dans leur position sociale à un moment
} 
L'ouvrage, déjà évoqué, de Vincent Debaene y insiste dans le chapitre « La littérature, les lettres et les sciences de l'homme » où il examine, méthodiquement, de Gustave Lanson à Georges Bataille, de Ramon Fernandez à André Breton, le sentiment de dépossession progressive de ces hommes de lettres chassés par ces sciences " positives », alors en plein essor, du territoire historique qui était celui de leurs prédécesseurs : l'étude de l'homme dans sa relation au monde. En réaction à ce constat, on peut avancer une réelle lucidité de la part de Lanson quant à la fonction, à venir, de la littérature, de l'art en général : celui de la présentation du personnage comme singularité quelconque. C'est dans un article intitulé « La littérature et la science », publié en 1895, qu'il prend acte de la dépossession de la littérature, par les sciences humaines, la sociologie, l'ethnologie, pour traiter de façon analytique la question du sujet, du « Qui ? ». Il tente alors de proposer un territoire spécifique, résiduel en quelque manière pour celle-ci, en la caractérisant par son objet spécifique : les « faits de conscience », « les passions, les sentiments, les instincts », ainsi que par son mode de transmission où la notion de vérité de type objectif n'est pas requise ${ }^{2}$.

C'est la perspective épistémologique dégagée par Foucault qui fait apparaître cette ligne de partage, une ligne de front bientôt, entre ce que Gisèle Sapiro appelle « les discours d'expertise » et ce que Deleuze appelle un « délire » : la littérature. En en faisant la question même de la modernité ; notre question, aujourd'hui encore. Foucault (1966) constate en effet...

... le seuil de notre modernité n'est pas situé au moment où on a voulu appliquer à l'étude de l'homme des méthodes objectives, mais bien le jour où s'est constitué un doublet empirico-transcendantal qu'on a appelé l'homme. [...] Comte et Marx sont bien témoins de ce fait que l'eschatologie (comme vérité à venir du discours sur l'homme) et le positivisme (comme vérité du discours définie à partir de celle de l'objet) sont archéologiquement indissociables : un discours qui se veut à la fois empirique et critique ; l'homme y apparaît comme une vérité à la fois réduite et promise. (Foucault $1966: 330-331$ )

Et cette conception, décisive, conquérante des sciences humaines, et des philosophies de l'histoire - conquérante car ce n'est que plus tard que se manifestera une auto-réflexivité critique sur l'objectivité de ces savoirs -, va écarter la littérature, l'art en général de leurs traditionnelles (depuis Aristote !) responsabi-

où les valeurs scientifiques s'affirment contre celles de la culture littéraire. Sous ce rapport, on peut voir dans les procès intentés aux écrivains naturalistes le signe de l'échec que rencontre Zola dans sa tentative de faire de la littérature un domaine d'expertise sociale à part entière. » (Sapiro 2011 : 691).

2 On trouvera une intéressante réflexion à ce sujet dans l'ouvrage de Jean Clair, sur l'évolution de l'art depuis le XIX ${ }^{e}$ siècle où il insiste sur « l'ab-normalité », sur « la fabrique du monstre dans l'art moderne » : Jean Clair, Hubris. La fabrique du monstre dans l'art moderne, Paris : Gallimard, coll. «Connaissance de l'inconscient », 2012. 
lités communes, peindre l'homme ou le passage, peindre la " nature humaine », « représenter » le sujet dans toutes ses figures par rapport à l'objet, au « milieu », à la société. Alors le littéraire s'emparera de la marge, des lieux-limites où ce savoir devient inopérant, en inventant des personnages comme figures d'exception, comme « hors-la-loi », mettant ainsi en question cette « vérité » réductrice de l'homme donnée comme objectivable ${ }^{3}$.

Les conséquences me paraissent importantes pour penser synthétiquement les orientations des pratiques esthétiques depuis maintenant plus de deux siècles. Je voudrai faire l'hypothèse suivante : l'art (la littérature en particulier) n'aura eu de cesse l'exploration de ce lieu conflictuel entre unicité de l'expérience et possibilités (largement problématiques) d'insertion de cette singularité dans une collectivité. En dernière instance, les sciences humaines tentent de définir la norme, la règle et les systèmes. Certes, et Foucault y insiste, la psychanalyse et l'anthropologie peuvent apparaitre comme des « contre-sciences » car elles problématisent les impensés qui président à ces cadres comportementaux en les historicisant et en les relativisant, faisant par là même la preuve de la finitude de l'homme, mais il n'en demeure pas moins qu'elles tentent, elles aussi, de rendre compte « scientifiquement » des comportements par une objectivation de l'inconscient (psychanalyse), par l'objectivation de tous les systèmes symboliques qui déterminent le rapport entre le sujet et le groupe auquel il s'identifie culturellement, fût-ce dans un phénomène paradoxal de rejet (anthropologie culturelle). On peut avancer alors que les pratiques esthétiques auront été le lieu de contestation de cette normativisation, de cette réglementation, de cette systématisation des expressions du Désir, de la Mort et de la Loi. Et elles l'auront fait en s'adossant de manière explicite ou implicite mais toujours conflictuelle, à la psychanalyse ainsi qu'à l'anthropologie. Des Surréalistes à Victor Segalen mais encore de Samuel Beckett, Michel Leiris, Desnos à Alain Robbe-Grillet et bien d'autres, la littérature s'inscrit, en creux, dans une volonté d'exploration des limites du champ des positivités que pointent réflexivement

\footnotetext{
${ }^{3}$ Foucault l'avait formulé clairement dans une perspective épistémologique : les positivités empiriques attestent l'acquisition d'un savoir analytique sur le langage, sur l'économie, sur la psychologie et fondent certes alors une connaissance « objective » de l'homme, mais une connaissance bornée par les présupposés de la représentation elle-même. Ces savoirs analytiques s'annexent cette représentation «bornée » et, dans le même temps, comme par réaction peut-être, les arts, la littérature tentent de s'affranchir de ces mêmes bornes. L'enjeu de cet essor des sciences humaines est précisé par Michel Foucault : il s'agit de donner à lire, à comprendre le « vécu » de l'homme. Mais l'analyse de ce « vécu » est elle-même prise dans le filet de ces positivités : qu'elle s'inscrive dans des pensées de type positiviste ou eschatologique (au premier rang desquelles le marxisme), qu'elle soit déterminée par des réflexions inspirées de la phénoménologie et donc de la connaissance originaire, spontanée de la nature qui s'esquisse avec le corps ; dans l'un et l'autre cas, ce vécu est inséré dans l'histoire d'une culture, dans une continuité évolutive ou dans une téléologie de l'histoire.
} 
ces deux contre-sciences ${ }^{4}$ mais sans la volonté de réduction par le « calcul », par le savoir « objectif $»^{5}$.

Probablement peut-on dater l'apparition du mot même de littérature de l'âge où se manifesta cette conscience adversative, différenciatrice, par rapport aux prétentions objectivantes des autres savoirs. Tout ceci justifie et radicalise la constatation de Proust lorsqu'il affirme, dans Contre Sainte-Beuve : « Les beaux livres sont écrits dans une sorte de langue étrangère ». Cette langue est étrangère à la communauté des discours sociaux, étrangère à la Loi (ou plutôt dit l'étrangeté inconnaissable de la Loi), étrangère à l'Histoire ou plutôt traite de ceux qui sont sans histoire, dont la parole est muette, rejetés qu'ils sont de l'Histoire des vainqueurs ${ }^{6}$.

\begin{abstract}
${ }^{4}$ C'est exactement l'analyse développée par l'avocat général Jean Boucheron lors du procès intenté, en 1958, à l'éditeur de l'Histoire de Juliette : Jean-Jacques Pauvert. Dans ses conclusions, comme le rappelle Gisèle Sapiro, celui-ci affirme que l'écrivain... « comme romancier il a le droit de traiter sous la forme du roman même des aberrations de l'homme, parce qu'il a le droit d'apporter sa contribution à la connaissance de l'homme, de révéler l'homme à lui-même. La sexualité n'est plus réservée à des ouvrages de pathologie ou à des études de morale. Liberté du thème et liberté du sujet, liberté de la forme d'expression, voilà ce que revendique le romancier et le poète. » (cité par Gisèle Sapiro, 2011 : 698).
\end{abstract}

${ }^{5}$ Il serait idéologiquement incohérent de ne pas remarquer, ici, que si cette opposition a pu advenir c'est que la société occidentale et son système de valeurs, ont évolué vers un ethos démocratique pour rendre possible l'expression de ces discours sur les limites, les marges qui ébranleront progressivement la centralité humaniste de son fondement. (Tout un discours serait à tenir sur la capacité, apparemment illimitée, d'intégration de la contradiction de la part du capitalisme " démocratique ».)

${ }^{6}$ Rétrospectivement, il me semble possible d'avancer que, tout au long du XIX ${ }^{\mathrm{e}}$ siècle, de Baudelaire à Poe, d'Hoffmann à Dostoïevski, non sans contrepartie « solaire » (ou réaliste, ou naturaliste), « fleurit » ce que ce fidèle entre tous de Hegel, Jürgen Habermas, appela une « littérature noire ». Et cette littérature noire, cette apparition du Mal comme catégorie esthétique a, me semble-t-il encore comme élément de caractérisation, l'emploi d'une langue dans la langue : une langue du Mal. Elle incarne d'abord le Mal parce que, d'elle, sourd un refus obstiné d'une finalité univoque, d'une téléologie historique. Hegel avait donc raison, de son point de vue, de détester le Romantisme en l'excluant de la sphère de l'« Esprit », comme expression du « négatif », en le plaçant dans la catégorie du Mal. Et s'il en est ainsi c'est que cette littérature se détourne du « vrai », du « solide », du « réel » et surtout du général puisque le personnage romantique est uniquement préoccupé de lui-même (voir Hegel (1979), chap. 3, « Dissolution de l'art classique »; cf la critique de Schlegel). Le Romantisme exacerbe en effet ce que Hegel déteste : la singularité comme figure de l'imprévisible (du point de vue de l'Histoire), de l'imprédictible (du point de vue de la question - anthropologique - du « Qui »). Encore convient-il de s'entendre sur le mot de « Mal»! Et pour ce faire, je suis au plus près la réflexion de Karl Heinz Bohrer dans son ouvrage Le présent absolu (Bohrer 2000) parce que cet ouvrage me paraît remarquable, parce que, surtout, il développe ce lien entre Mal et dissolution, contrainte ou choisie, d'un imaginaire du temps « orienté » et orienté, dans l'ontologie naturaliste occidentale, selon l'idée du progrès, sous toutes ses formes. Il suffit en effet de faire se mouvoir la roue herméneutique d'un tour supplémentaire pour avancer le lien entre l'illisibilité du comportement humain, le devenir énigme de la question du «Qui ? » et dénégation du temps historique comme téléologie. À partir du XIX ${ }^{e}$ siècle et comme le note justement, je crois, Böhrer, « le mal » est avant tout ici un attribut de l'intemporalité, c'est-à-dire de l'élimination des conditions spatiotemporelles de notre faculté de jugement normale, qui détermine aussi les critères moraux. 
Une conscience adversative : elle apparaît donc avec le Romantisme, tout particulièrement allemand. Le Romantisme va installer, durablement, une conception de l'esthétique où le concept de réalité échappe au rationalisme hégélien pour caractériser, précisément, « ce qui résiste au sujet» (Hans Blumenberg). Cette littérature prône encore l'informe, l'inachevé, le devenir, la métamorphose, au lieu de l'être. L'" inconscient esthétique » fait apparaître en effet un univers de présences inquiétantes, d'allusions à tout ce qui échappe : « des mécanismes sombres, des déterminations sans figure, tout un paysage d'ombre que directement ou indirectement on a appelé l'inconscient » (Foucault 1966 : 337).

Une esthétique littéraire du Mal se définit comme un mode de présentation fictionnel de comportements énigmatiques, suggérant une imagination incontrôlée, échappant à la formalisation précisément et en cela irréductible à la raison solaire comme Réponse (non sans rapports étroits avec ce sublime que Burke définissait selon les trois termes de « terreur », d'« obscurité » et d'« infini »). Walter Benjamin, il y a bien longtemps, avait clairement pointé cette évolution de la littérature, romanesque dans son propos, comme la mise en scène de figures de l'exception, de la marginalité, dans l'article (Benjamin 1930) qu'il consacrait, sous le titre « La crise du roman » à Döblin et à son ouvrage Berlin Alexanderplatz en opposant la choralité épique du conte à l'expression romanesque de l'être seul, de celui à qui, pour paraphraser une notation de Michaux, « il reste peu de temps pour aller au bout de lui-même » :

Le romancier, quant à lui, s'est isolé du peuple et de ses faits et gestes. Le lieu de naissance du roman est l'individu dans sa solitude, qui ne dispose plus d'expression exemplaire de ses intérêts les plus vitaux et qui, n'étant conseillé par personne, est lui-même incapable de conseiller qui que ce soit. Écrire un roman c'est pousser l'incommensurable dans la représentation de l'existence humaine à son extrême. (Benjamin 1930 : 189)

«S'isoler du peuple et de ses faits et gestes » et ce sera la perte du sens du geste, allégorisée par Kafka ; " pousser l'incommensurable dans la représentation de l'existence humaine » et ce sera une littérature de la perte de la relation, de la dissolution de la conscience comme intériorité et de la volonté individuelle comme conditions d'une exposition lisible du conflit et, donc, comme possibilité d'inscription de l'agir dans le temps historique (très exactement, répétons-le, ce que Blumenberg qualifie de conception contemporaine de la « réalité » objective et subjective : « ce qui résiste au sujet»).

Cette « perte du sens du geste » est peut-être l'indicateur le plus manifeste du conflit entre sciences exactes, sciences humaines et littérature. Dans un essai intitulé « Notes sur le geste » Giorgio Agamben (2002) en faisait le constat significatif. Alors qu'avec le développement de la technique photographique, les 
scientifiques à la fin du XIX $X^{\mathrm{e}}$ siècle, analysent les gestes en les décomposant (Muybridge, à l'Université de Pennsylvanie photographie «L'homme qui marche »), objectivant en cela le petit opuscule de Balzac formulant de façon plus facétieuse que scientifique sa Théorie de la démarche, il note que, au moment même où apparaît l'image-mouvement du cinématographe, « ... la bourgeoisie qui était encore quelques dizaines d'années auparavant, solidement assurée de la possession de ses symboles, succombe à l'intériorité et se livre à la psychologie » (Agamben 2002 : 63) comme conséquence réflexive de la perte, anthropologique, idéologique, politique du sens de ses gestes. Quant à l'anthropologie, il est évident que sa recherche porte, constamment, sur l'explicitation du sens des gestes, des comportements symboliques, des rites, des règles déterminant les échanges, etc. Kafka apparaît alors bien comme le révélateur génial de la fonction même de la littérature : une problématisation énigmatique du sens du geste. Cette épistémologie des rapports entre sciences positives et littérature et tout particulièrement le rapport complexe, tendu, fasciné avec l'anthropologie car cette science humaine étudie, précisément, l'homme dans la double dynamique d'identification et de relation qui détermine sa vie en société aura donc fait la preuve d'une « querelle de territoires » qui rend sans doute compte, a posteriori, de cette tendance forte du littéraire, jusqu'à la fin $\mathrm{du} \mathrm{xx}^{\mathrm{e}}$ siècle, à se déporter vers la présentation de sujets/personnages comme expressions des marges, comme « singularités quelconques »... La littérature «moderne » depuis Sade et jusqu'à Bataille, Deleuze ${ }^{7}$ et bien d'autres, aura tenté de donner à percevoir tout ce qui, chez le sujet échappe à une réduction rationalisante, sans restes, creusant par là un espace adversatif, mettant en évidence les limites mêmes de la représentation comme achèvement ${ }^{8}$.

Sans doute, et parce que ce sujet mériterait une réflexion autonome qu'il n'est guère possible de conduire ici, faudrait-il relire toute la littérature « expérimentale » de l'immédiat après Seconde Guerre mondiale en France, celle du Nouveau Roman et, d'ailleurs les thèses de la « Nouvelle critique » qui lui ont été associées, en termes anthropologiques, orientations de lectures à quoi l'époque ne se prêtait guère, dominée par une affirmation, trop vite devenue formule : celle de « la mort de l'homme». Certes, Bruce Morrissette dès 1963 concentrait, à rebours des grilles de lectures alors dominantes, ses réflexions (mais de façon vraiment

\footnotetext{
${ }^{7}$ « La littérature est délire, et à ce titre joue son destin entre deux pôles du délire. Le délire est une maladie, la maladie par excellence, chaque fois qu'il érige une race prétendument pure et dominante. Mais il est la mesure de la santé quand il invoque cette race bâtarde opprimée qui ne cesse de s'agiter sous les dominations, de résister à tout ce qui l'écrase et l'emprisonne et de se dessiner en creux dans la littérature comme processus. » (Deleuze 1993 : 15)

${ }^{8}$ Il serait ici possible de proposer un raisonnement pas aussi digressif que son éloignement pourrait le donner à croire, celui de la sur-représentation comme expression des totalitarismes. De fait, la représentation aura toujours, historiquement été l'expression d'une littérature « majeure » et, à ce titre, liée aux idéologies dominantes : tous les fascismes du $\mathrm{Xx}^{\mathrm{e}}$ siècle ont valorisé sans partages cette représentation.
} 
très " psychologique ») sur la signification anthropologique des romans d'Alain Robbe-Grillet (Morrissette 1963), signification qu'évoquait au demeurant, mais ici encore de manière vraiment très superficielle Roland Barthes dans sa préface ${ }^{9}$; certes les liens entre déconstruction de la fiction traditionnelle et déterminismes anthropologiques étaient évoqués par ce même Robbe-Grillet toujours dans son essai Pour un Nouveau Roman, mais on peut rapidement dire quels rapports entre discours anthropologiques et littérature auront été, non absents, bien au contraire, mais occultés surtout par le champ critique, pendant toute la seconde moitié du Xx siècle. Il serait souhaitable, voire nécessaire, de relire, aujourd'hui les œuvres de Butor, de Simon, de Duras et de bien d'autres en mettant en évidence ce dialogue silencieux, occulte entre perspectives anthropologique et poétiques.

Mais qu'en est-il depuis le début de ce millénaire (ou un peu avant) des rapports entre littérature et anthropologie ? Je m'inspirerai, pour tenter de répondre, de quatre auteurs, apparemment très dissemblables, de deux philosophes, Giorgio Agamben et Gilbert Simondon, d'une sociologue de formation littéraire, Marielle Macé déjà citée et, enfin, d'un critique littéraire, Dominique Rabaté.

C'est dans son bref essai récent, intitulé Désirs de disparaître. Une traversée du roman français contemporain (Rabaté 2015) que ce dernier se demande comment la littérature française actuelle hérite, de façon critique, des « débats théoriques » des années 60/70 sur le statut de la fiction notamment romanesque et de la conception idéaliste d'un Absolu littéraire et du « langage à l'infini ».

La période actuelle me semble marquer une inflexion différente. J'y vois une réouverture de l'univers fictionnel, où le jeu reprend ses droits, où la machine romanesque se remet à fonctionner, en faisant précisément du motif de la disparition son moteur dramatique. (Rabaté 2015 : 40)

Rabaté repère, en effet, nombre de romans incluant comme thème principal celui de la disparition du personnage. Il ajoute, à la suite de Pierre Zaoui et en faisant de celui-ci un héritier de Blanchot que ce motif thématique de la disparition s'accompagne en général, chez le narrateur, d'une volonté de " discrétion ». Il cite Pierre Zaoui : «Peindre, écrire, peut-être filmer, ce serait toujours chercher à disparaître » (Zaoui 2013 : 124). Selon trois acceptions : disparition de l'auteur, de l'œuvre au profit de l'exercice ou de la tentative incessante, disparition de l'art lui-même.

Je retiens de cette première référence : le retour de la fiction, le thème de la disparition (ou de l'évanouissement) du personnage, la « discrétion » de l'instance narrative ${ }^{10}$.

\footnotetext{
${ }_{9}^{9}$ Dans la préface, Barthes note : « ... provoquer le sens pour l'arrêter, ce n'est rien d'autre que de prolonger une expérience moderne qui a son origine dans l'activité surréaliste et qui engage l'être même de la littérature, c'est-à-dire en définitive la fonction anthropologique qu'elle détient au sein de la société historique tout entière. » (Morrissette 1963 : 11).

${ }^{10}$ Quelques titres significatifs à cet égard : Tanguy Viel, La disparition de Jim Sullivan, Sylvie
} 
De l'ouvrage de Marielle Macé, déjà cité, je souhaite faire mienne l'idée que les arts contemporains (cinéma, littérature, bandes dessinées etc.) rendent compte avec insistance d'une forme documentaire, d'une volonté de « documentation des formes de vie ». Cet essai tente de caractériser la vie comme forme, comme formes pour définir ce que pourrait être une « stylistique de l'existence », mais de la manière la moins prescriptive qui soit (contrairement à l'exigence d'évaluation que ne peut délaisser totalement la sociologie, fût-elle marquée par le principe du doute systématique, de l'incertitude... ${ }^{11}$ ). Une forme de vie doit, constamment, s'éprouver, se remettre en cause, en exiger une ou des autres, se pluraliser donc, en contestant l'exigence idéologique de la société d'une forme de vie surdéterminée par notre rôle, de notre place, de notre fonction dans la société où nous vivons ${ }^{12}$.

Et il me semble en effet que nombre de fictions contemporaines, profondément anthropologiques en cela, s'efforcent de décrire les formes de vie, de vies contraintes, de « vies nues », de vies « pauvres en mondes » comme nos formes d'existence les plus fréquentes aujourd'hui. Loin de se situer dans la continuité fictionnelle des grands romans du XIX ${ }^{e}$ siècle ces fictions traitent de la vie quotidienne la plus quelconque, développent des intrigues minimales, sans recours à quelque événement dramatique, tragique ou, moins encore, héroïque comme moteur diégétique. Elles documentent les formes de vie communes, elles décrivent nos « lieux-communs », ceux dont la formulation rythme nos rapports à autrui, voire à nous-mêmes.

C'est là faire un constat que Giorgio Agamben - largement cité d'ailleurs par Marielle Macé - effectuait mais à propos de la vision du monde franciscaine lorsqu'il rappelait le dualisme fondamental de toute forme de vie humaine. C'est en effet dans L'usage des corps, Homo sacer, IV, 2, dans le chapitre " Pour une ontologie du style » que se trouve formulée cette opposition :

Germain, Hors champ mais aussi cette disparition du personnage est fréquente dans les romans de Modiano, Ndiaye, Quignard ou Garcin...

11 J'avoue apprécier tout particulièrement dans les réflexions de Marielle Macé, l'attestation constante de son cheminement réflexif avec la pensée des autres, et dans le cas présent avec celle de Luc Boltanski, auquel elle rend un hommage appuyé, précisément à cause de sa volonté de laisser dans l'analyse sociologique la place au doute, à l'incertitude, contrairement à la pensée « judiciaire » de Bourdieu :

« J'admire de ce point de vue le parcours irrésolu, mécontent, et pourtant constamment convaincu de Boltanski dans ces questions, en l'occurrence le fait qu'il semble n'en avoir jamais fini avec ce tourment, et la façon dont, de livre en livre, se re-débat pour lui l'écart, qui est donc aussi un lien, entre description et jugement. Je retiens de sa démarche, tantôt allant vers, tantôt s'éloignant de la critique, la place concrète faite à l'incertitude. » (Macé $2016: 290)$

${ }_{12}$ L'expression de « vie nue » est employée par Agamben pour qualifier la situation limite de l'« Homo sacer » : «Il est important de ne pas confondre la vie nue avec la vie naturelle. Par sa division et sa capture dans le dispositif de l'exception, la vie prend la forme de la vie nue, c'est-àdire d'une vie qui a été scindée et séparée de sa forme. » (Agamben $2015: 359$ ). 
Les théologiens distinguent entre la vie que nous vivons (vita quam vivimus), c'est-à-dire l'ensemble des faits et des événements qui constituent notre biographie, et la vie par laquelle nous vivons (vita qua vivimus), ce qui rend la vie vivable et lui donne un sens et une forme... [...] dans toute existence, ces deux vies se présentent divisées et, toutefois, on peut dire que toute existence est la tentative, souvent manquée et néanmoins sans cesse réitérée, pour réaliser leur coïncidence. En effet, seule une vie où cette scission disparaît est une vie heureuse. (Agamben $2015: 359)$

Et il fait de cette opposition un thème essentiel du roman depuis Henry James, le « laboratoire » même de la question des formes de vie : « ... le lieu où la recherche de la coïncidence entre les deux vies a trouvé son laboratoire le plus sophistiqué est le roman moderne » (Agamben 2015 : 313).

D’Annie Ernaux à François Bon, de Modiano à Sebald, du dernier Calvino (Palomar) à Bergounioux nombre de fictions de ce début du $\mathrm{Xx}^{\mathrm{e}}$ siècle documentent ces vies que nous vivons comme des vies souvent privées de choix, privées de « ce qui rend vivable la vie ». Le personnage qui disparaît ne fait, dans ce geste d'autosuppression, d'auto-exclusion que vérifier la radicalité de l'opposition entre « la-vie-que-l'on-vit » et « la vie-par-laquelle-on-vit » en supprimant la première au profit, dans le meilleur des cas, de la seconde.

Et ce faisant, cette littérature s'avère constituer un geste profondément politique en dénonçant l'imposition d'une vie nue, en mettant en évidence la façon dont nos vies se débattent pour essayer de se constituer comme valeur. Cette volonté de faire comparaître les formes de vie me semble aussi une tendance significative de la fiction aujourd'hui.

\section{Anthropologie du fait littéraire : conclusion}

Georges Simondon enfin. Pour rendre compte de cette tendance de la fiction dans laquelle la perspective anthropologique me paraît essentielle, il faut sans doute en passer par une référence à la société civile, aujourd'hui, ou, du moins au rapport entre individualisation et collectivité. C'est à Georges Simondon - tel en tout cas qu'il est commenté par Paolo Virno -, que je dois cette approche du mode de constitution de l'individualité, de la singularité (Virno 2002). Loin de s'opposer à l'individualité, la « multitude », les manifestations collectives de la société civile (qui sont sans doute une marque de notre temps) contribuent à son façonnement. Simondon définit le sujet individuel comme un champ de tensions entre un pré-individuel (le general intellect et les rapports de productions dominants, la langue, les perceptions sensibles spontanées) et les traits spécifiques d'une individuation faisant la singularité de chacun. 
Si le « je » individué cohabite avec le fond biologique de l'espèce (la perception sensorielle, etc.), avec les caractères publics ou inter-psychiques de la langue maternelle, avec la coopération productive et le general intellect, il faut ajouter cependant, que cette cohabitation n'est pas toujours pacifique et qu'elle engendre même toutes sortes de crises. Le sujet est un champ de bataille. Il n'est pas rare que les aspects pré-individuels semblent mettre en question l'individuation : cette dernière se révèle être un résultat précaire, toujours réversible. (Virno $2002: 84-85$ )

Le sujet, champ de bataille. Peut-être le rapport entre littérature et anthropologie, depuis le XIX ${ }^{\mathrm{e}}$ siècle, se trouve-t-il alors défini. Mais tandis que la littérature des siècles passés mettait en fiction une opposition fondée sur la singularité sans partages du « héros » (ou de l'héroïne) qui entre en conflit avec la société à cause même de son exception qualitative, de René de Chateaubriand jusqu'à Des Esseintes de Huysmans en passant par Emma Bovary ou Raskolnikov, chacun de ces personnages ayant un ancêtre commun, en terme de singularité adversative, dans l'inénarrable Don Quichotte de la Manche bien sûr, la littérature depuis une trentaine d'années maintenant traite de cette «bataille » avec des singularités quelconques, des personnages du commun qui se définissent, précisément, par rapport à la «multitude », à la société civile à laquelle ils appartiennent dans leur quotidienneté la plus répétitive.

\section{Références bibliographiques}

Agamben 2002 : G. Agamben, « Notes sur le geste», trad. de l'italien par Daniel Loayza, in Moyens sans fins : notes sur la politique, Paris : Éditions Payot \& Rivages, coll. « Rivages poche. Petite bibliothèque », 59-71.

Agamben 2015 : G. Agamben, L'usage des corps, Homo sacer, IV, 2, traduit de l'italien par J. Gayraud, Paris : Éditions du Seuil, coll. «L'ordre philosophique ».

Bataille 1956 : G. Bataille, « Un livre humain, un grand livre », Critique n 105, 99-112. Benjamin 2000 [1930] : W. Benjamin, « La crise du roman. À propos de Berlin Alexanderplatz de Döblin », in W. Benjamin, Euvres II, trad. de l'allemand par M. de Gandillac, R. Rochlitz et P. Rusch, Paris : Gallimard, coll. « Folio. Essais », 189197. Première publication : Die Gesellschaft $\mathrm{n}^{\circ} 7,1930$.

Bohrer $2000: \mathrm{K}$. H. Bohrer, Le présent absolu : du temps et du mal comme catégories esthétiques, trad. de l'allemand par O. Mannoni, Paris : Éditions de la Maison des sciences de l'homme.

Debaene 2010 : V. Debaene, L'adieu au voyage. L'ethnologie française entre science et littérature, Paris : Gallimard, coll. «Bibliothèque des sciences humaines ».

Deleuze 1993 : G. Deleuze, Critique et clinique, Paris : Les Éditions de Minuit, coll. «Paradoxe».

Foucault 1966 : M. Foucault, Les mots et les choses, une archéologie des sciences humaines, Paris : Gallimard, coll. «Bibliothèque des sciences humaines ». 
Hegel 1979 : G. W. F. Hegel, Esthétique II, trad. de l'allemand par S. Jankélévitch, Paris : Flammarion, coll. « Champs. Champ philosophique ».

Hugo 1842 : V. Hugo, Le Rhin II, Paris : Henzel.

Macé 2016 : M. Macé, Styles. Critique de nos formes de vie, Paris : Gallimard, coll. « NRF essais ».

Morrissette 1963 : B. Morrissette, Les romans de Robbe-Grillet, préface de Roland Barthes, Paris : Éditions de Minuit, coll. «Arguments ».

Rabaté 2015 : D. Rabaté, Désirs de disparaître. Une traversée du roman français contemporain, Université du Québec à Rimouski : Tangence éditeur, coll. "Confluences ».

Robbe-Grillet 1963 : A. Robbe-Grillet, Pour un nouveau roman, Paris : Éditions de Minuit. On se reportera à l'article « Nature, humanisme, tragédie », 45-69.

Sapiro 2011 : G. Sapiro, La responsabilité de l'écrivain. Littérature, droit et morale en France (XIX ${ }^{e}-X X I^{e}$ siècle), Paris : Éditions du Seuil.

Virno 2002 : P. Virno, Grammaire de la multitude. Pour une analyse des formes de vie contemporaines, trad. de l'italien par Véronique Dassas, Nîmes : Éditions de l'éclat, Montréal : Conjonctures.

Zaoui 2013 : P. Zaoui, La discrétion ou l'art de disparaître, Paris : Autrement, coll. « Les grands mots $»$.

\section{Филип Дарос}

\section{Антрополошки дискурс и књижевност}

Деловање књижевности можемо да дефинишемо као промишљање питања идентитета историјског субјекта, што у фикцији бива представљено у виду лика, његовог деловања, његовог односа према месту, времену, његових симболичких поступака, а то би одговарало начину на који савремена антропологија приступа идентитету, посматрајући га кроз идентификацију и релацију. Такво антрополошко схватање књижевног испитаћемо из перспективе књижевне критике.

Кључне речи: књижевност, антропологија, друштвене науке, књижевна критика, субјекат, идентитет. 